\title{
Neurosurgical Interventions in Children with Maroteaux-Lamy Syndrome
}

\author{
Case Report and Review of the Literature
}

\author{
V.I. Vougioukas ${ }^{a}$ A. Berlis ${ }^{b}$ M.V. Kopp ${ }^{c}$ R. Korinthenberg ${ }^{c} \quad J_{\text {Spreer }}^{b}$ \\ V. van Velthoven ${ }^{a}$ \\ Departments of a Neurosurgery, ${ }^{b}$ Neuroradiology and ${ }^{c}$ Neuropediatrics, University of Freiburg, Germany
}

\section{Introduction}

Mucopolysaccharidoses (MPS) constitute a group of diseases in which the storage of lipids in neurons and of polysaccharides in connective tissues results in a conjunction of neurological and skeletal abnormalities that are virtually unique. The nervous system may also be involved secondarily through hyperplasia of connective tissue. This can lead to obliteration of the subarachnoid space and consequently hydrocephalus or to dural thickening resulting in spinal neurological manifestations.
Patients with Maroteaux-Lamy syndrome [MPS type 6 (MPS6)] have a defect of the enzyme arylsulfatase B. Intelligence is normal but severe skeletal deformities are present. The neurological manifestations are not sharply delineated. There are reports of patients with MPS6 who presented with hydrocephalus communicans, pseudotumor cerebri and empty sella syndrome as well as cervical and lumbar spine disease [1-5]. We report the case of a 14-year-old patient with Maroteaux-Lamy syndrome who was initially treated elsewhere for compressive damage of the optic nerves and subsequently for hydrocephalus communicans and who presented to our service with progressive tetraparesis secondary to craniocervical stenosis and ligamentum flavum hypertrophy. The case is presented as a basis for discussion of the mechanisms of neurological manifestations in patients suffering from this rare metabolic disorder.

\section{Case Report}

A 14-year-old female patient was admitted for evaluation of progressive tetraparesis. She was diagnosed as having MPS6 (Maroteaux-Lamy syndrome) at the age of 2. Initial investigations included urine metabolite screening that showed abnormal excretion of dermatan and heparan sulfate. All the clinical signs, including typical facies of Maroteaux-Lamy syndrome (large upper eyelid, hyperplastic lips, head tilted backward) and dwarfism, were present. The child had been doing well until the age of 8 , when she reported visual impairment. The ophthalmological examination in another hospital showed full-thickness corneal clouding in the right eye and moderate

Vassilios I. Vougioukas, MD

Albert-Ludwigs University of Freiburg, Department of Neurosurgery

Breisacherstrasse 64

D-79106 Freiburg im Breisgau (Germany)

Tel. +49 761270 5006, Fax +49 761270 5090, E-Mail vvougio@nz.ukl.uni-freiburg.de 


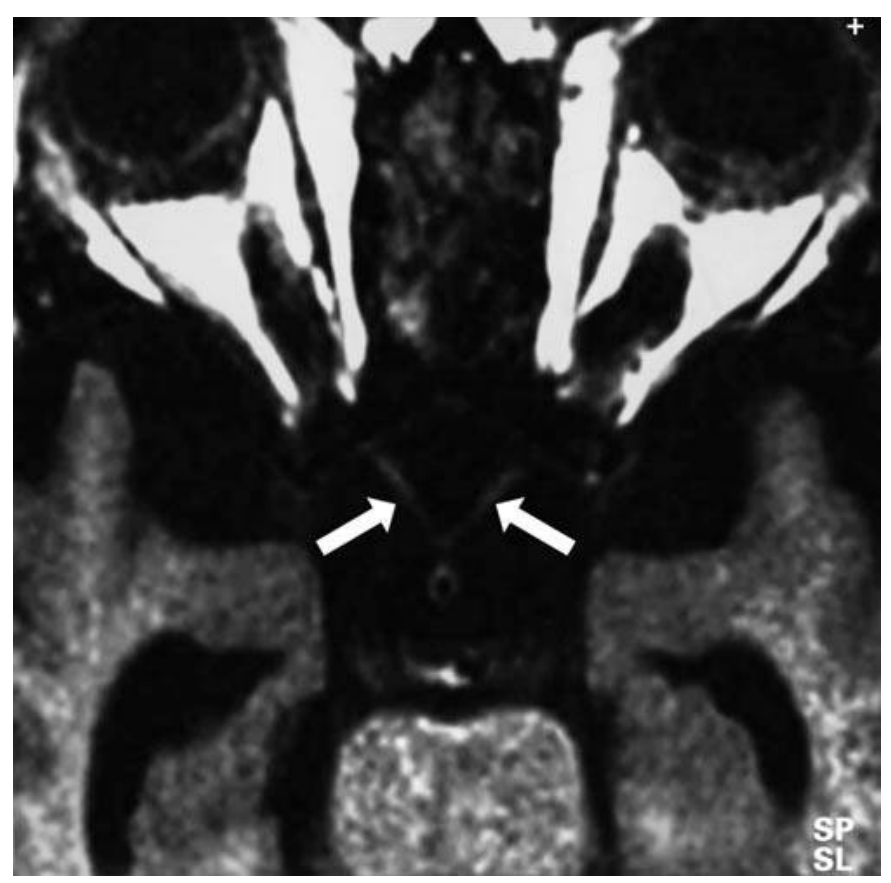

Fig. 1. A preoperative axial T1-weighted image demonstrating bilateral atrophy of the intradural part of the optic nerve (arrows) and large lipid deposits within the orbit.

clouding in the left eye. A penetrating keratoplasty was performed on the right eye. Visual acuity improved and the patient did well until the age of 12, when she was admitted again for progressive visual loss. Based on the findings of the ophthalmological examination, it was decided to perform a keratoplasty on the left eye. Nine months after surgery, visual loss was still progressive. Fundus examination revealed bilateral optic atrophy. A cranial magnetic resonance imaging (MRI) examination showed optic canal stenosis and bilateral optic nerve atrophy (fig. 1). Due to disease progression, craniotomy and bilateral optic nerve decompression by enlargement of the optic canal was performed. The patient initially benefited from the procedure, but the postoperative course was complicated by respiratory insufficiency and a permanent tracheostomy had to be placed.

Five months later, the patient had to be hospitalized again because of headaches and drowsiness. A cranial computed tomographic scan (CCT) revealed moderate symmetrical ventriculomegaly with prominent temporal horns and widening of the subarachnoid space compared to a study that had been done 6 months earlier. A spinal puncture revealed a pressure of $16 \mathrm{~cm} \mathrm{H}_{2} \mathrm{O}$. The patient underwent a ventriculoperitoneal shunt with a favorable outcome.

The clinical course was stable until the patient reached the age of 14 , when she was admitted to the neuropediatric service because of progressive tetraparesis. Six weeks before admission, her arms and legs became increasingly spastic. Finally, she was unable to walk, elevate her arms or use her hands for eating and drinking. Physical examination showed, beside the typical signs of Maroteaux-Lamy syndrome, a spastic tetraparesis which was greater in the legs. MRI studies of the craniocervical junction showed a multisegmental stenosis of the upper cervical spine and marked flavum hypertrophy but no signs of cervical myelopathy (fig. 2). Laminectomy from $\mathrm{C} 1$ to $\mathrm{C} 3$ and consecutive enlargement of the foramen magnum was performed (fig. 3). Promptly after decompression of the bony canal stenosis and removal of the thickened flavum ligament, the dura started pulsating and appeared normal. The postoperative course was favorable. There was considerable reduction of spasticity and the patient started regaining motor control of all four extremities. Two months after the operation, she was able to walk without support for 20-30 meters, raise her arms and hands to head level and feed herself independently.

\section{Discussion}

Patients with Maroteaux-Lamy syndrome may present with various neurological symptoms. Disease pathology, as in almost every case of MPS, is attributed to lipid accumulation in the CNS and the musculoskeletal system. The present case is of particular interest because of the insidious development of neurological symptoms and the dramatic improvement after neurosurgical interventions.

The reported patient initially presented elsewhere with sudden visual impairment. In patients with MPS6, corneal clouding develops in the first decade of life [5]. There are several reports of cases that had to be treated with corneal transplantation due to progressive visual loss [6, 7]. Papilledema and optic atrophy may also occur $[8,9]$. The pathophysiology of optic atrophy remains unclear. An autopsy study suggested that lipid deposition in the dura of the optic nerve sheath is responsible for optic nerve damage in this patient population [9]. Neglected hydrocephalus may also accelerate the progression of visual loss in patients with MPS. In cases where examination of visual acuity and fundoscopy fail to provide a sufficient explanation for disease progression, a CCT and MRI studies should be performed to rule out hydrocephalus and optic nerve compression. In the case reported here, MRI images showed large lipid deposits surrounding the intraorbital and intradural part of the optic nerve. Despite a normal appearance of the bony canal, the optic nerve was bilaterally atrophied (fig. 3). Because of progressive visual loss, $8 \mathrm{~mm}$ of the bony optic canal were removed and the falciform fold was incised. Although the postoperative course was complicated by respiratory insufficiency, the patient finally benefited from the procedure, since postoperatively, visual acuity improved.

The cause of hydrocephalus in patients with MPS, although it constitutes a well-known entity in such cases, remains controversial. There have been several reports of hydrocephalus communicans in children with Hurler's syndrome (MPS1), Sanfilippo's syndrome (MPS3) and 
Fig. 2. Sagittal $\mathrm{T} 2$-weighted images of the upper cervical spine and the craniocervical junction performed preoperatively in 1997 (A) and 1999 (B). Note the effect of the craniocervical decompression as well as disease progression in the postoperative images $(\mathbf{C})$. The fourth ventricle is completely filled with lipid deposits.
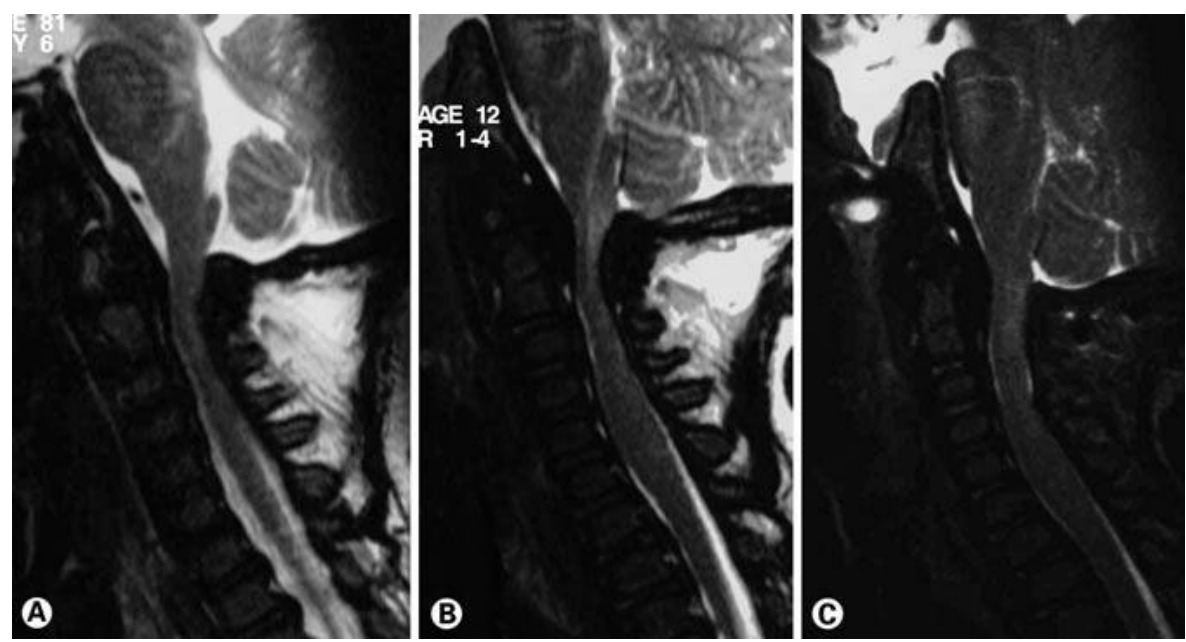

Maroteaux-Lamy syndrome (MPS6) [1, 10, 11]. Patients may present with signs of raised intracranial pressure, macrocephaly, behavioral disturbance or visual impairment due to optic atrophy. It is postulated that storage of mucopolysaccharides and infiltration by PAS-positive phagocytes into the arachnoidal villi result in a significant reduction in cerebrospinal fluid reabsorption with consequent ventricular enlargement [1,12]. Fowler et al. [13] proposed that infiltration of the leptomeninges with mucopolysaccharide material might cause obliteration of the cranial subarachnoid space. In most cases, a ventriculoperitoneal shunt leads to rapid clinical improvement. Placement of a ventriculoatrial shunt in cases of Maroteaux-Lamy syndrome should be avoided when possible, because cardiac disease is relatively common [14].

Infiltration of the pachymeninges, which is also quite common in children suffering from storage diseases, may result in an obliteration of the subarachnoid space over the cerebral convexity or spinal cord compression. Electron microscopic studies of the thickened cervical dura in a patient with Maroteaux-Lamy syndrome revealed numerous chondrocyte-like balloon cells that contained stacked membranes [3]. Spinal cord compression due to dural or ligament hypertrophy and bony stenosis should always be kept in mind in children with MPS6 [3]. Myelopathy may also be secondary to atlantoaxial dislocation, resulting in instability and developmental anomalies of the odontoid process [15]. Interestingly, our patient had degenerative changes of the atlantoaxial joint and a normal odontoid process (fig. 3). Spinal cord compression is not restricted to the cervical level. The lumbar spine
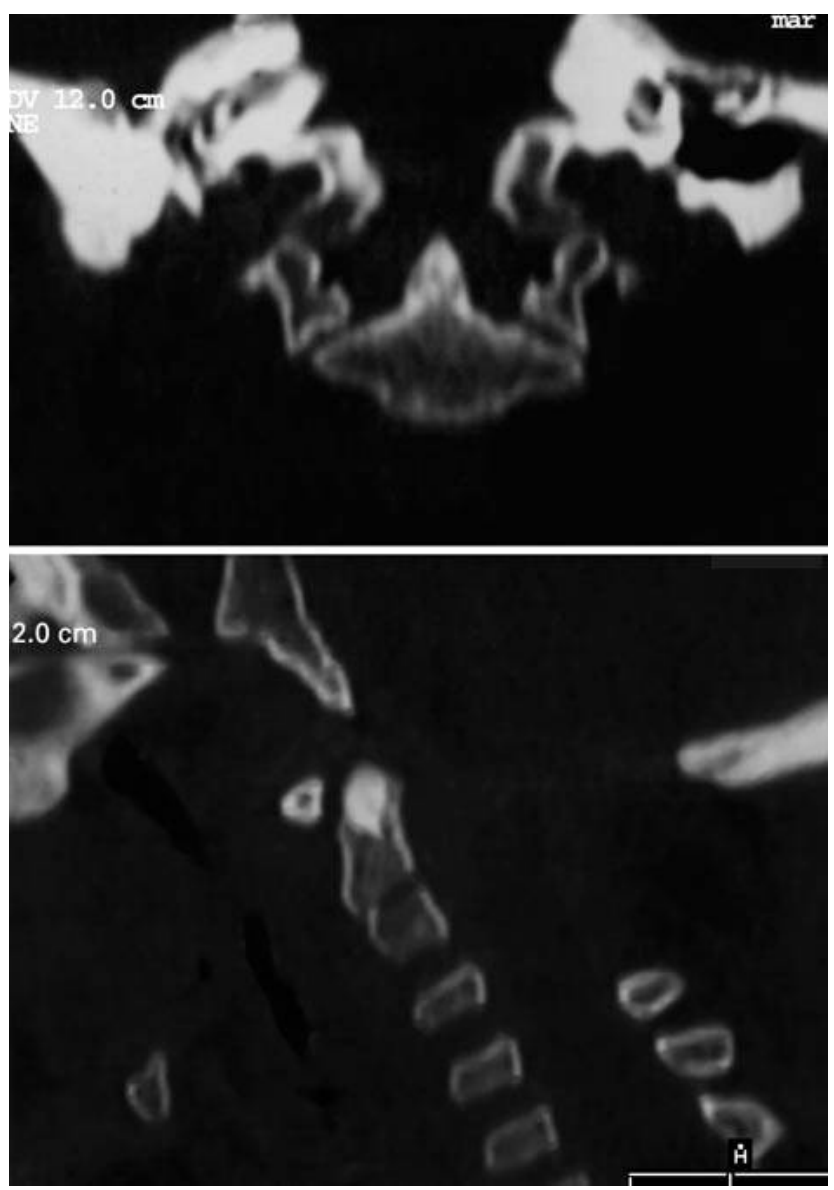

Fig. 3. Postoperative $\mathrm{CT}$ images of the craniocervical junction and the atlantoaxial joint. Note the degenerative changes in the right atlantoaxial joint. 
cluding laminectomy and removal of the thickened dura when needed is the treatment of choice [3]. Disease progression may be insidious and life threatening [17]. In the case reported here, the child developed a complete tetraparesis and dissociated sensory loss within 6 weeks. Interestingly, although the MRI images showed an abnormal thickening of the pachymeningeal structures, the dura appeared to be macroscopically normal. Therefore, and considering the extent of lipid deposition, we decided intraoperatively not to incise the dura. Nevertheless, bony stenosis and flavum hypertrophy were very prominent. The rapid onset of clinical improvement after the performance of a decompressive laminectomy was very impressive. Postoperative images suggest that the patient actual- ly benefited more from the enlargement of the foramen magnum than from the laminectomy.

The clinical course of patients with MPS6 can be complicated by a number of neurological conditions. The difficulty of making the right diagnosis promptly is often underestimated. Since patients with MPS6 are usually not mentally retarded, a simple procedure may essentially contribute to improvement in the quality of life. Hydrocephalus, optic nerve compression and spinal myelopathy should always be kept in mind in order to avoid severe complications such as irreversible visual loss, herniation and tetraparesis. A neurosurgical intervention, when indicated, is essential for the adequate treatment of patients suffering from this metabolic disorder.

\section{References}

1 Sheridan M, Johnston I: Hydrocephalus and pseudotumour cerebri in the mucopolysaccharidoses. Childs Nerv Syst 1994;10:148-150.

2 Goldberg MF, Scott CI, McKusick VA: Hydrocephalus and papilledema in the MaroteauxLamy syndrome (mucopolysaccharidosis type VI). Am J Ophthalmol 1970;69:969-975.

3 Young R, Keinman G, Ojemann RG, Kolodny E, Davis K, Halperin J, Zalneraitis E, DeLong GR: Compressive myelopathy in MaroteauxLamy syndrome: Clinical and pathological findings. Ann Neurol 1980;8:336-340.

4 Von Muhlendahl KE, Bradac GB: Empty sella syndrome in a boy with mucopolysaccharidoses type VI (Maroteaux-Lamy). Helv Paediatr Acta 1975;25:185-190.

5 Suveges I: Histological and ultrastructural studies of the cornea in Maroteaux-Lamy syndrome. Graefes Arch Clin Exp Ophthalmol 1979;212:29-39.
6 Varssano D, Cohen EJ, Nelson LB, Eagle RC Jr: Corneal transplantation in the MaroteauxLamy syndrome. Arch Ophthalmol 1997;115: 428-429.

7 Orgul S, Daicker B, Kain HL: Simultaneous corneal transplantation in mucopolysaccharidosis (in German). Klin Monatsbl Augenheilkd 1991;198:430-432.

8 Naumann GO, Rummelt V: Clearing of the para-transplant host cornea after perforating keratoplasty in Maroteaux-Lamy syndrome (type VI-A mucopolysaccharidosis) (in German). Klin Monatsbl Augenheilkd 1993;203: 351-360.

9 Sturmer J: Type VI-A mucopolysaccharidosis (Maroteaux-Lamy disease). Clinico-pathologic case report (in German). Klin Monatsbl Augenheilkd 1989;194:273-281.

10 Robertson SP, Klug GL, Rogers JG: Cerebrospinal fluid shunts in the management of behavioural problems in Sanfilippo syndrome (MPS III). Eur J Pediatr 1998;157:653-655.
11 Schwartz GP, Cohen EJ: Hydrocephalus in Maroteaux-Lamy syndrome. Arch Ophthalmol 1998;116:400.

12 Van Aerde J, Campbell A: Hydrocephalus and shunt placement. Am J Dis Child 1983;137: 187-188.

13 Fowler GW, Sukoff M, Hamilton A, Williams JP: Communicating hydrocephalus in children with inborn errors of metabolism. Childs Brain 1975;1:251-254.

14 Muenzer J: Mucopolysaccharidoses. Adv Pediatr 1986;33:269-302.

15 Kennedy P, Swash M, Dean MF: Cervical cord compression in mucopolysaccharidosis. Dev Med Child Neurol 1973;15:194-199.

16 Sheridan M, Chaseling RC, Johnston I: Hydrocephalus, lumbar canal stenosis and Maroteaux-Lamy syndrome (mucopolysaccharidosis type 6). Case report. J Neurosurg Sci 1992;36: 215-217.

17 Peterson DI, Bacchus H, Seaich L, Kelly TE: Myelopathy associated with Maroteaux-Lamy syndrome. Arch Neurol 1975;32:127-129. 